\title{
Discovery of radio-loud quasars with redshifts above 4 from the PMN sample $e^{\star, \star \star}$
}

\author{
I. M. Hook ${ }^{1, \star \star \star}$, R. G. McMahon ${ }^{2}$, P. A. Shaver ${ }^{3}$, and I. A. G. Snellen ${ }^{4}$ \\ 1 Department of Physics, University of Oxford, Nuclear \& Astrophysics Laboratory, Keble Road, Oxford OX1 3RH, UK \\ e-mail: imh@astro.ox.ac.uk \\ 2 Institute of Astronomy, Madingley Road, Cambridge CB3 OHA, UK \\ 3 European Southern Observatory, Karl Schwarzschild Straße 2, 85748 Garching b. München, Germany \\ ${ }^{4}$ Institute for Astronomy, Royal Observatory, Edinburgh, EH9 3HJ, UK
}

Received 5 February 2002 / Accepted 11 June 2002

\begin{abstract}
We present results of a new, large survey for high-redshift radio-loud quasars, which targets quasars with $z>4$. The survey is based on the PMN and NVSS radio surveys, optically identified using digitised UKST $B, R$ and $I$ plates. Six new $z>4$ flat-spectrum QSOs have been discovered, and one previously known $z>4$ QSO rediscovered, based on their red optical colours. The QSOs discovered in this survey are bright in both radio and optical bands; in particular PMN J1451-1512 $(z=4.763, \mathrm{I}=17.3, R=19.1)$ and PMN J0324-2918 $(z=4.630, R=18.7)$ are very luminous. PMN J1451-1512 at $z=4.763$ is also now the most distant radio-selected quasar. In addition, 9 new quasars with $3.5<z<4.0$ were discovered during the survey. We present spectra and finding charts for the new quasars. We also derive a surface density of $1.0 \pm 0.4 \times 10^{-3} \mathrm{sq} \mathrm{deg}^{-1}$ for flat-spectrum $z>4$ QSOs with $S \geq 72$ mJy and $R<21$ mag.
\end{abstract}

Key words. quasars: general

\section{Introduction}

Radio selection remains one of the most efficient ways of finding high-redshift AGN. This approach has the further advantage of being less prone to selection effects than optical selection, since radio emission is unaffected by either intrinsic or extrinsic absorption due to dust. The specific aim of this work was to find optically bright, radio-selected high-redshift quasars. These can be used for unbiased studies of damped Lyman alpha systems at high redshift and other follow-up studies such as searches for associated high-redshift galaxy clusters.

We therefore began to carry out a large, systematic survey aimed specifically at $z>4$ QSOs. Our method involves the optical identification of flat-spectrum radio sources and the spectroscopic follow-up of the red stellar identifications. This approach exploits the fact that quasars at high redshift have redder optical colours than their low-redshift counterparts due to

Send offprint requests to: I. M. Hook, e-mail: ihook@gemini . edu

$\star$ The work presented here is based in part on data obtained with the ESO facilities on La Silla (EFOSC/3.6-m).

$\star \star$ Figures 7 and 8 are only available in the electronic form at http://www. edpsciences.org

$\star \star \star$ Present address: Gemini Observatory, 670 N. A'ohoku Place, Hilo, HI 96720, USA. absorption by intervening HI (see Fig. 1 in Hook et al. 1995), and has proved successful at finding high-redshift quasars in the past (Hook et al. 1995, 1996, 1998).

Previous work using well-defined quasar samples has shown that $z>4$ radio-loud quasars are likely to be rare objects, both because the quasar population as a whole appears to decline at redshifts above 2-3 (Kennefick et al. 1996; Hawkins \& Veron 1996; Schmidt et al. 1995; Warren et al. 1995) and because radio-loud quasars represent only about $10 \%$ of the full quasar population.

Specific studies of the radio-loud quasar population have shown that these objects are indeed rare at $z>4$. Dunlop \& Peacock (1990) presented strong evidence for a drop in the space density of radio-loud quasars between $z=2$ and $z \approx 3$ based on radio-selected samples reaching $S_{2.7 \mathrm{GHz}}=100 \mathrm{mJy}$. More recently, significant progress has been made towards understanding the evolution of the radio-loud quasar population out to $z \sim 4$ and the potential effects of absorption by dust, by the study of a completely identified, large area, flat-spectrum radio sample with $S_{2.7 \mathrm{GHz}} \geq 250 \mathrm{mJy}$ (Shaver et al. 1996; Wall et al., in preparation). The low numbers of high-redshift quasars found in these studies demonstrates that there is a distinct drop-off in the space density of quasars at $z>3$. Thus for our new survey to be successful it must reach fainter radio flux density limits than the above surveys (to sample further down 
the luminosity function), and cover a significant fraction of the sky.

Here we present the first results of this new survey for highredshift radio-loud quasars. As will be seen in Sect. 2, the survey uses deeper radio and optical data than previous radio-loud quasar surveys, and covers a very large area in the Southern sky ( $\sim 7500$ sq deg, comparable to that of the planned 10000 sq deg of the Sloan survey). Our survey has produced seven $z>4$ flatspectrum quasars, one of which was previously known. The survey complements the survey of Snellen et al. (2001) which contains four flat-spectrum $z>4$ in the Northern sky, selected using a similar method.

\section{The radio sample and the optical identification procedure}

The parent radio sample used in this study is based on the Parkes-MIT-NRAO radio survey (PMN, Griffith et al. 1995 and references therin), selected at $5 \mathrm{GHz}$. The data cover the southern sky with $\delta<10^{\circ}$ to a flux density limit of 20-72 mJy depending on declination. No additional flux density limit was applied when carrying out the survey, but note that when considering the statistics of the final quasar sample, we consider sources with $S \geq 72 \mathrm{mJy}$, since the PMN completeness at lower flux density levels is patchy (see Fig. 1).

To provide accurate positions and spectral index information, the PMN catalogue was matched to the $1.4 \mathrm{GHz}$ NRAOVLA Sky Survey (NVSS, Condon et al. 1998), which covers the declination range $\delta \geq-40^{\circ}$. All NVSS sources matching within a 2 arcmin radius of a PMN position were kept.

Since the beam sizes of the PMN and NVSS surveys are different (4.2' for PMN and 45" for NVSS), the 1.4 flux density used to determine spectral indices were calculated by summing the total flux density from all NVSS sources whose positions were within a 2 arcmin radius of the PMN position. Flat spectrum objects with $S_{\text {PMN }} \geq 50$ mJy were then selected using the criterion $\alpha_{1.4}^{5} \geq-0.5$ where $S \propto v^{\alpha}$. This gave 5976 PMN radio sources.

The NVSS positions were matched to optical catalogs which were derived from scans of UK Schmidt Telescope (UKST) plates. The scans were produced using the Automatic Plate Measuring (APM) facility (McMahon \& Irwin 1992). The optical identification procedure was similar to that used in the past to make POSS-based identifications of radio samples, described in Hook et al. (1995, 1996, 1998). However the current survey also makes use of I-band plates for the first time.

The plate data used is from the UKST survey of the southern sky in the $B, R$ and $I$ bands, reaching limiting magnitudes of approximately $22.6,21.0$ and $19.5 \mathrm{mag}$ respectively. The blue plates are in the $B_{\mathrm{J}}$ passband, 3950-5400 $\AA$, and have 606 centres spaced at intervals of 5 degrees. In most cases the matching $R$ plate used was from the OR survey with a passband of 5950-6900 $\AA$, although in some fields not yet covered by the OR survey, the old $R$ plate was used (6300-6900 $\AA$ ). The $I$ plates used IV-N emulsion + RG715 filter giving a passband of 7150-8900 ̊.

The maximum redshift of quasars that our survey can detect in principle is defined by the redshift at which the Ly $-\alpha$ line is redshifted out of the redder passbands. For the $R$-plates this occurs at redshifts above $z=4.7$ and for the $I$ plates at redshifts above $z=6.3$.

\subsection{Area covered by the survey}

Figure 1 shows the spatial distribution of the radio sources which form the basis of the survey. These lie in the region of overlap of the PMN and NVSS surveys. The effective area of our quasar survey is defined by the subset of this region that is covered by APM scans of UKST plates.

All the available $B, R$ and $I$ plates have now been scanned in the region of overlap with our radio sample $\left(-40^{\circ}<\delta<+2.5^{\circ}\right.$, avoiding the galactic plane, $\left.|b|>30^{\circ}\right)$. At the time of the spectroscopic observations reported here, an area of $7525 \mathrm{sq} \mathrm{deg}$ was covered by plates in the $B$ and $R$ bands (4637.5 sq deg in the South Galactic Cap region and $2887.5 \mathrm{sq}$ deg in the North Galactic Cap region). A total of $3887.5 \mathrm{sq} \mathrm{deg}$ also had I-band plates. The spatial distribution of the plates is shown in Fig. 2. Of this, two regions around RA $=13 \mathrm{~h}, \mathrm{DEC}=0^{\circ}$ have no radio data, as shown in Fig. 1. The total area of overlap with the $B, R$ data is $7265.5 \mathrm{sq} \mathrm{deg}$ of which $4637.5 \mathrm{sq} \mathrm{deg}$ is in the South Galactic Cap (SGC) region and $2628.0 \mathrm{sq}$ deg is in the North Galactic Cap (NGC). Of this a total of $3637.5 \mathrm{sq}$ deg also had $I$ data.

In addition there are small areas within the region of radio/optical overlap that were not yet covered by the NVSS survey at the time our QSO survey was carried out. This resulted in some PMN sources not being matched with an NVSS counterpart in certain regions, as can be seen in Fig. 2. When considering the statistics of our final quasar sample, we take this incompleteness into account in a statistical way by calculating the fraction of PMN sources with $S_{\mathrm{PMN}}>72 \mathrm{mJy}$ that do not have NVSS counterparts. In the SGC the matched fraction is 0.955 and in the NGC it is 0.871 .

\subsection{Selection of the spectroscopic sample}

Optical identifications were made based on positional coincidence of the NVSS position with an optical counterpart on the UKST plates. A matching radius of 3.0" between the NVSS and optical positions was used (see Fig. 3 for the distribution of positional differences).

From these identifications, red, stellar objects were selected for spectroscopic follow up. Figure 4 shows colour-magnitude and colour-colour diagrams for optical identifications within $3^{\prime \prime}$ of the NVSS position. The first spectroscopic sample contained any stellar object with $B-R \geq 1.5$. A second sample, the " $I$ band sample", was then defined, which contained stellar objects with $R-I \geq 1.0$, or $B-I \geq 2.0$ that were not already included in the first sample. The criterion for being considered a stellar object was $\sigma_{\text {class }} \leq 3.0$ where $\sigma_{\text {class }}$ is the APM classification parameter measured from the $R$ plate. If the object was not detected in $R$ then the $I$ plate classification was used. These selection criteria resulted in a sample of 228 sources. Of these, 33 had known redshifts from the literature prior to the start of this project. 


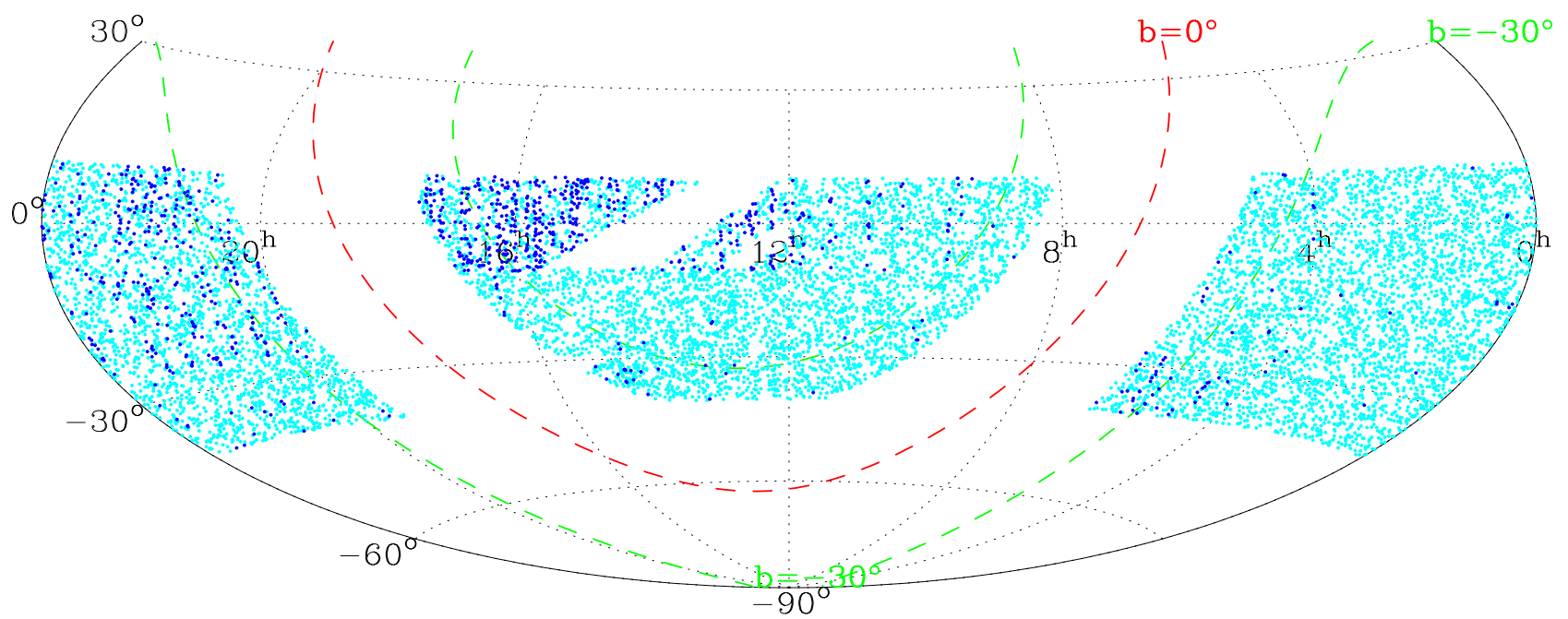

Fig. 1. Area covered by the radio data at the time of the survey. light dots show PMN sources with $S \geq 72$ mJy which had a counterpart in the NVSS catalogue, and dark dots show those PMN sources which did not have an NVSS counterpart. Usually this was because the NVSS survey had not been completed in those areas at the time (March 1998).

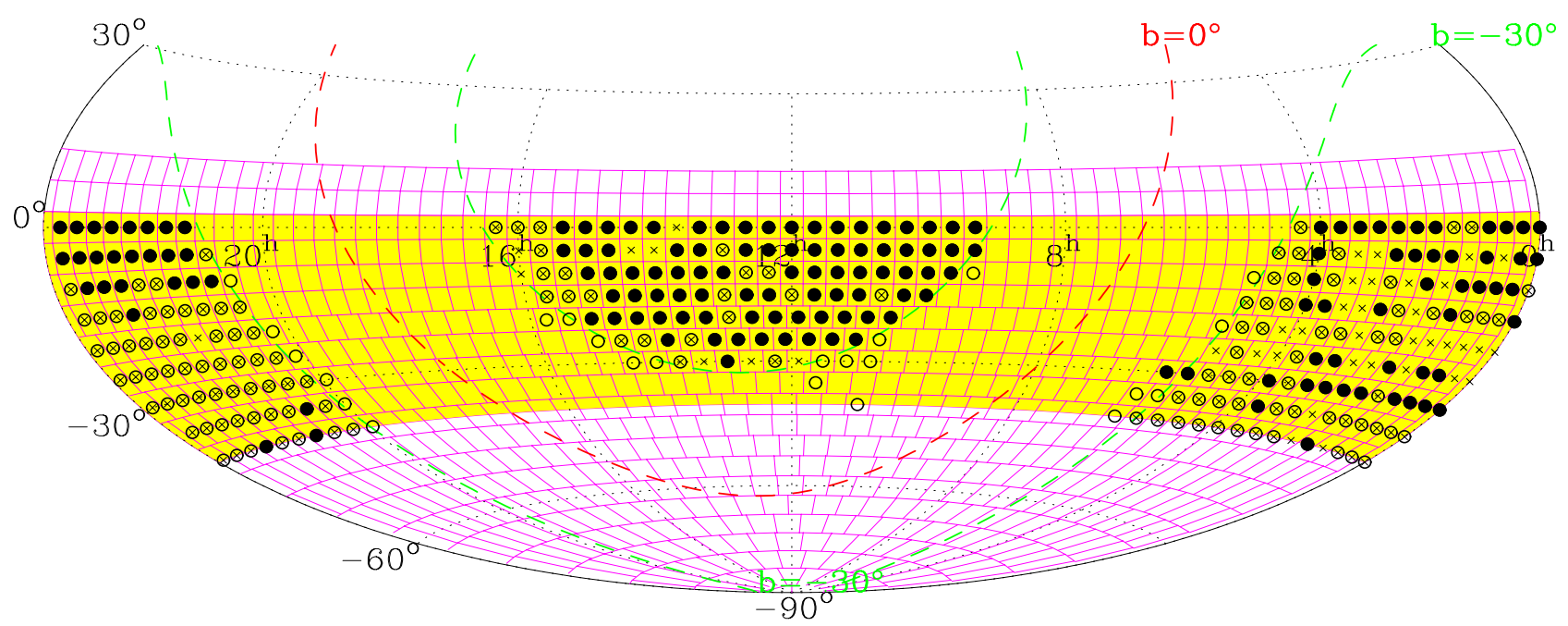

Fig. 2. Area covered by the optical data. Crosses show UKST fields whose centres satisfy $|b|>30 \mathrm{deg},-40 \mathrm{deg}<\delta<0$ deg, open circles show fields that have $B$ and $R$ plates, and filled circles show fields that have $B, R$ and $I$ plates. A total of 313 fields have at least $B$ and $R$ plates available, corresponding to an area of $7525 \mathrm{sq} \mathrm{deg}$. The shaded area represents the declination range of the survey, defined by the overlap of the NVSS, PMN and UKST data.

\section{Spectroscopic observations and results}

In total, 169 candidates were observed spectroscopically. During two runs in October 1998 and May 1999, 157 objects were observed at the ESO $3.6 \mathrm{~m}$, La Silla, Chile using the EFOSC spectrograph. For both runs the detector used was “ccd40", a Loral CCD with $2048 \times 2048$ pixels, binned $2 \times 2$. For the 1999 May observations grism \#12 was used, covering the spectral range $5800 \AA-9500 \AA$ with a dispersion of $4.23 \AA$ per binned pixel. For the 1998 October observations grisms \#4 and \#5 were used covering the ranges 3420-7460 $\AA$ and 6000$10000 \AA$ respectively with dispersions of 4.08 and $4.27 \AA$ per binned pixel. The slit width was chosen to match the seeing, typically $1.2-1.5$ arcsec. For the 1998 October observations a sky PA of $270^{\circ}$ was used, and for the 1999 May observations the slit was at the paralactic angle. Approximate relative flux calibration was obtained using observations of spectrophotometric standards from Hamuy et al. (1994). Twelve sources were observed at AAT using the RGO spectrograph in October 1996.

26 sources remain to be observed, of which 24 are in the North Galactic Cap (NGC) and 2 are in the South Galactic Cap (SGC) region.

The data were reduced using standard procedures with the IRAF $^{1}$ software environment.

${ }^{1}$ IRAF is distributed by the National Optical Astronomy Observatories, which is operated by the Association of Universities for Research in Astronomy, Inc. (AURA) under cooperative agreement with the National Science Foundation. 
Table 1. Summary of current survey status for sources with $S \geq 50 \mathrm{mJy}$.

\begin{tabular}{lrccc}
\hline \hline \multicolumn{1}{c}{$\begin{array}{c}\text { Number of candidates } \\
\text { with } z \text { data }\end{array}$} & & $3.5<z<4.0$ & $4.0<z<4.5$ & $z>4.5$ \\
\hline Present observations & 169 & 7 & 4 & 2 \\
Literature data & 33 & 2 & 1 & 0 \\
\hline Total & 202 & 9 & 5 & 2 \\
\hline
\end{tabular}

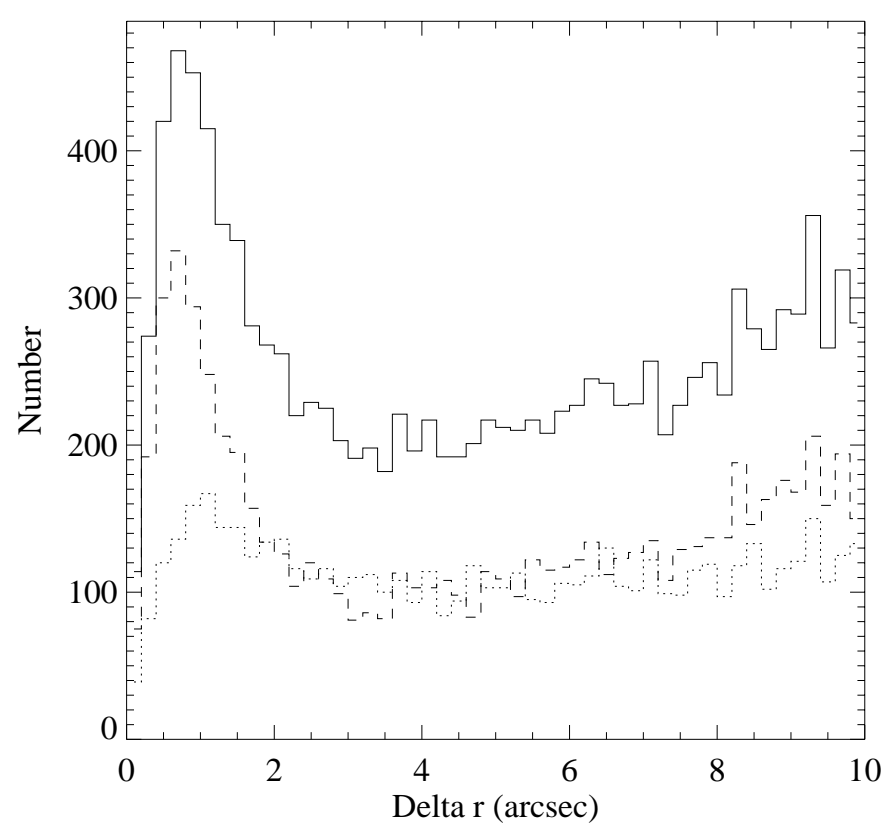

Fig. 3. Distribution of differences between the optical (APM) and radio (NVSS) positions. The solid line is the histogram for all sources, the dashed line is for objects classified as stars and the dotted line is for objects classified as galaxies. A matching radius of 3 arcsec was used in this survey.

Table 1 summarises the current survey status and in Table 2 we give redshifts, optical magnitudes and radio flux densities for the objects which were identified as new high-redshift quasars. The spectra are shown in Fig. 5, and finding charts, with J2000 coordinates, are given in Fig. 7. Two of the $z>4$ QSOs are discussed in more detail below.

PMNJ1451-1512: this is now the most distant radio-selected quasar. The object is blank on the UKST $B$-band plate, detected in $R$, and very bright in $I$ (17.3). Near-IR magnitudes from the 2MASS survey are $J=16.39 \pm 0.10, H=15.28 \pm 0.09, K=$ $14.67 \pm 0.09$.

The optical spectrum (Fig. 5) shows strong lines of Ly $\alpha$ SiIV/OIV and CIV from which we estimate a redshift of 4.763 . However the absorption of the CIV and Ly- $\alpha$ line make redshift determination uncertain. A $4800 \mathrm{~s}$ IR spectrum was obtained using SOFI at the $3.5 \mathrm{~m}$ NTT, La Silla on 31 May 1999. The spectrum shows strong, broad emission lines of CIII and $\mathrm{MgII}$ at a redshift of 4.764 and approximately 4.78 respectively (the MgII line has a complex shape, see Fig. 6). We adopt a redshift of 4.763 for this QSO.

The NVSS map (see Fig. 8) shows a source at the optical position of the QSO, and a second NVSS source about 4 arcmin away. The PMN position appears to lie between the two, although much closer to the first position (that of the QSO). It is possible that the PMN flux density is overestimated because of a contribution from the second source.

PMN J0525-3343: this QSO was discovered in the AAT run of October 1996. It was reobserved at the Las Campanas 100" $\mathrm{du}$ Pont telescope using the ModSpec spectrograph and this spectrum is shown Fig. 5. The somewhat lower signal-to-noise combined with the lack of clean strong lines make the redshift determination difficult. However Péroux et al. (2001) have since obtained a higher quality spectrum from which a more accurate redshift measurement can be made. They obtain $z=$ $4.383 \pm 0.034$ assuming rest wavelengths for the emission lines that have not been corrected for systematic shifts, or $z=4.338$ when assuming the rest wavelengths used in Table 3, which have been corrected for systematic shifts as described by Tytler \& Fan (1992).

This source has been confirmed as an X-ray source with ROSAT HRI and its X-ray properties are the subject of another paper (Fabian et al. 2001).

\section{Discussion}

By using radio and optical multicolour data covering a significant fraction of the sky, we have produced a sample of highredshift, optically bright radio-loud quasars.

Since this new quasar sample is well-defined, we can use it to estimate the surface density of $z>4$ quasars. To do this we consider objects with radio flux densities above the brightest limit of the PMN surveys, $72 \mathrm{mJy}$. The SGC region of our survey has a high completeness of spectroscopic follow up (only two objects, or $1 \%$, were not observed in this region) and also had a high completeness of the NVSS survey (0.955). There are four $z>4$ QSOs in the SGC region of the complete sample, which implies a surface density of $0.92 \pm 0.5 \times 10^{-3} \mathrm{sq} \mathrm{deg}^{-1}$ for $z>4$ QSOs with $S \geq 72 \mathrm{mJy}$.

If the whole survey is considered, and the completeness for the SGC and NGC are taken as $94 \%$ and $61 \%$ respectively (as implied by the number of spectroscopically observed candidates in each region combined with the NVSS completeness factors derived in Sect. 2) then the derived surface density is 
Table 2. Summary of optical and radio properties for quasars with $z>3$ in the sample. A "-" indicates that no data were available. The positions are the APM optical positions determined from the $R$ plates.

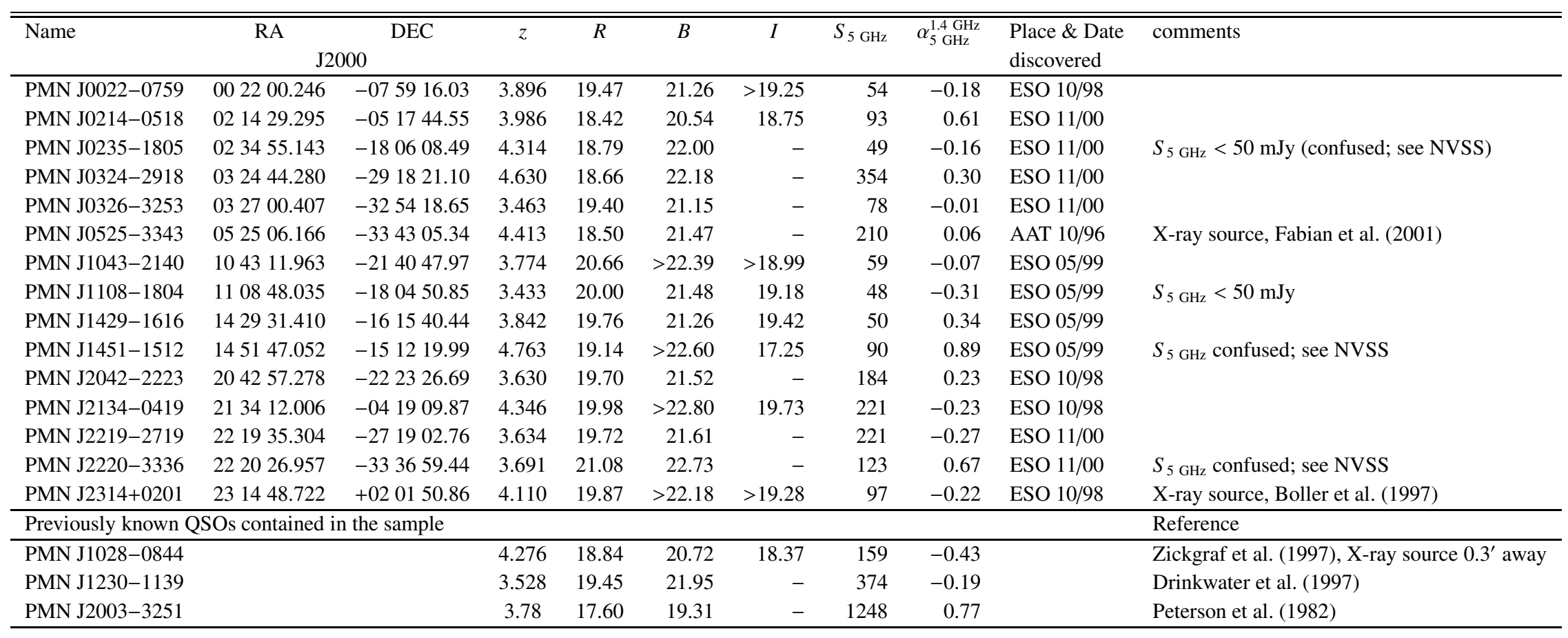



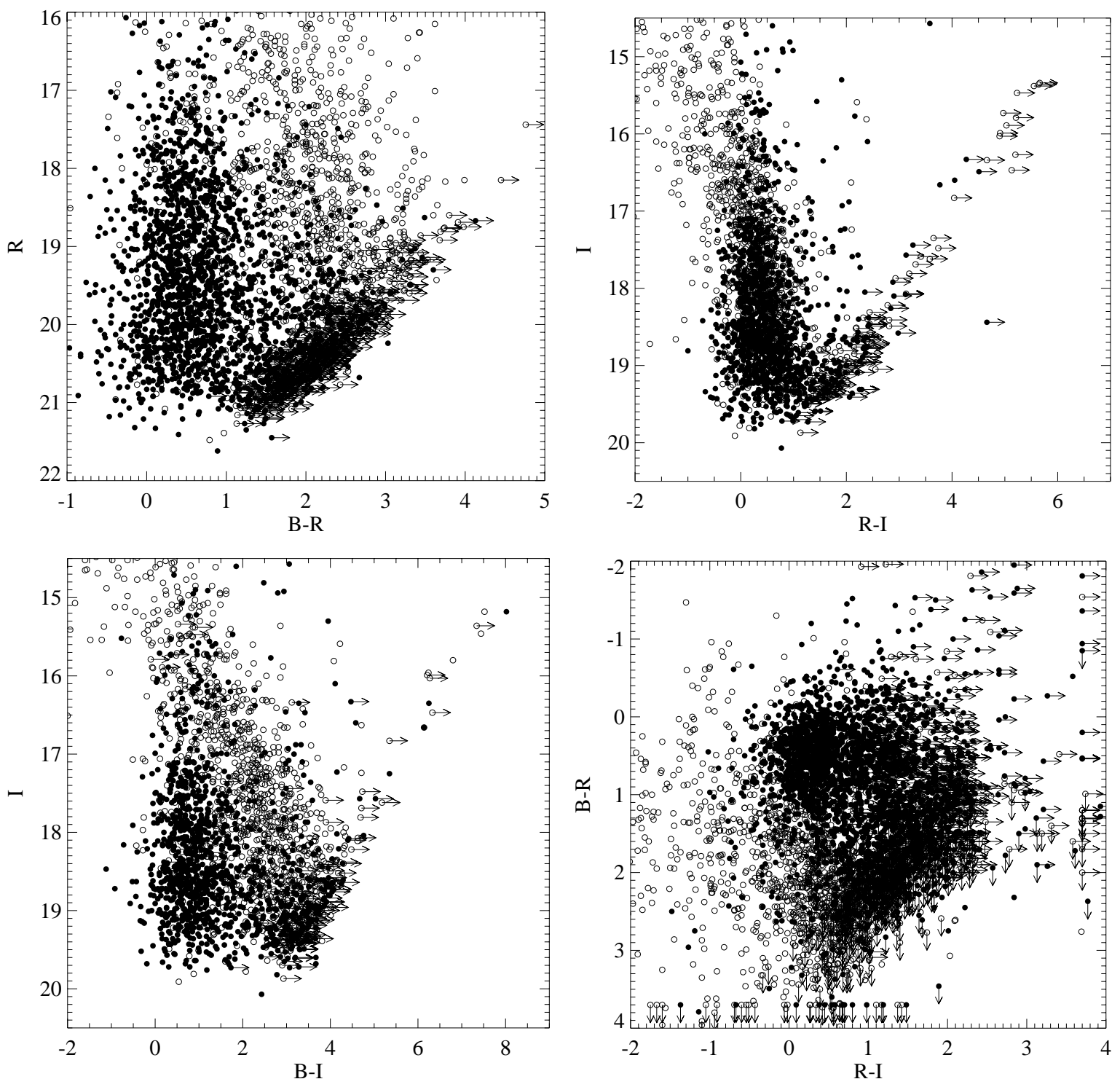

Fig. 4. Colour-magnitude and colour-colour plots for the APM identifications within $3^{\prime \prime}$ of the NVSS radio position. Solid symbols represent objects classified as stellar, open symbols represent objects classified as galaxies by the APM. Arrows are used to represent limits on the colours when objects are not detected in one or more of the three passbands.

$1.0 \pm 0.4 \times 10^{-3} \mathrm{sq} \mathrm{deg}^{-1}$. This is fully consistent with the value determined from the SGC alone.

This value is similar to that of 1 per $1600 \mathrm{sq} \mathrm{deg}(6.3 \times$ $10^{-4}$ ) found by Snellen et al. (2001) using a similar technique although with a slightly different radio and optical flux density limits $(R=20$ compared to $R=21$ for the current survey, $S \geq 30$ mJy compared to $S>72 \mathrm{mJy}$, an upper redshift limit defined by the red optical filter of $z \sim 4.5$ rather than $\sim 4.7$, and a radio spectral index cut at -0.35 rather than -0.5 ).

There were no quasars found in our survey with $z>4.76$ despite the fact that bright quasars with $4.9 \lesssim z \lesssim 6.3$ should have been detectable on the I-plates. Allowing for the $86 \%$ completeness of spectroscopic follow up of the I-band sample, the effective area covered was $2930 \mathrm{sq} \mathrm{deg}$. Therefore we derive an upper limit of $3.4 \times 10^{-4} \mathrm{sq} \mathrm{deg}^{-1}$ for the surface density of flat spectrum quasars with $I<19.5, S_{5 \mathrm{GHz}} \geq 25 \mathrm{mJy}$ and $4.9 \lesssim z \lesssim 6.3$.
Finally, the new sample of quasars presented in this paper represent some of the most luminous objects in the Universe and may also represent extreme peaks in the matter density distribution at high-redshift. They are therefore ideal targets for various follow-up programs such as high-redshift absorption line studies and searches for associated high-redshift clusters.

Acknowledgements. RGM thanks the Royal Society for support. We thank Jason Spyromilio for providing the IR spectrum of PMN J14511512.

We also thank Mike Irwin and the staff at the APM facility in Cambridge for producing the scans of UKST plates used in this survey.

This publication makes use of data products from the Two Micron All Sky Survey, which is a joint project of the University of Massachusetts and the Infrared Processing and Analysis Center, funded by the National Aeronautics and Space Administration and the National Science Foundation. 
PMN J0022-0759

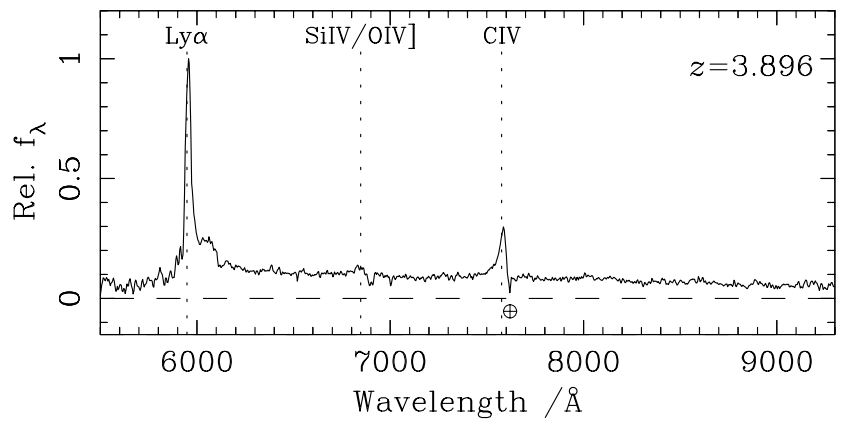

PMN J0214-0518

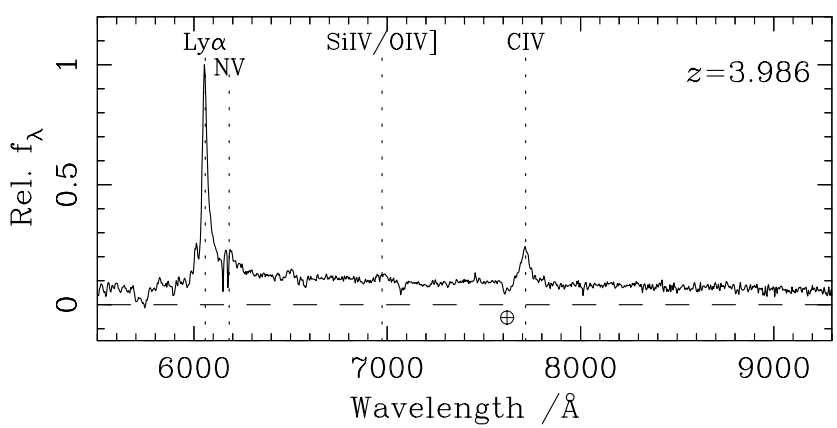

PMN J0235-1805

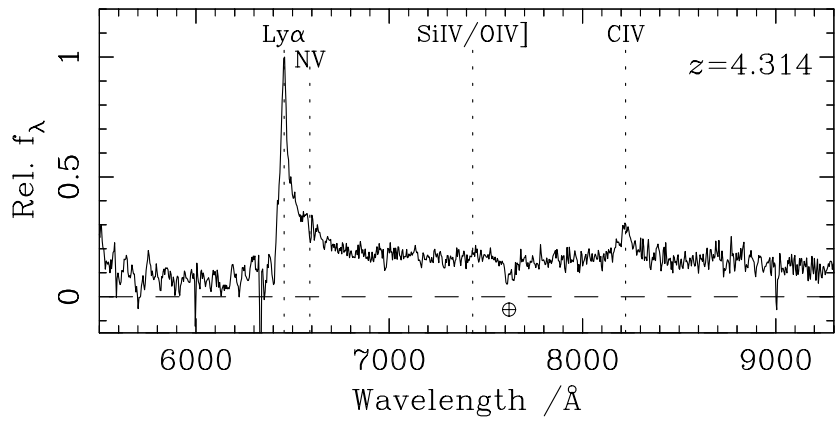

PMN J0324-2918

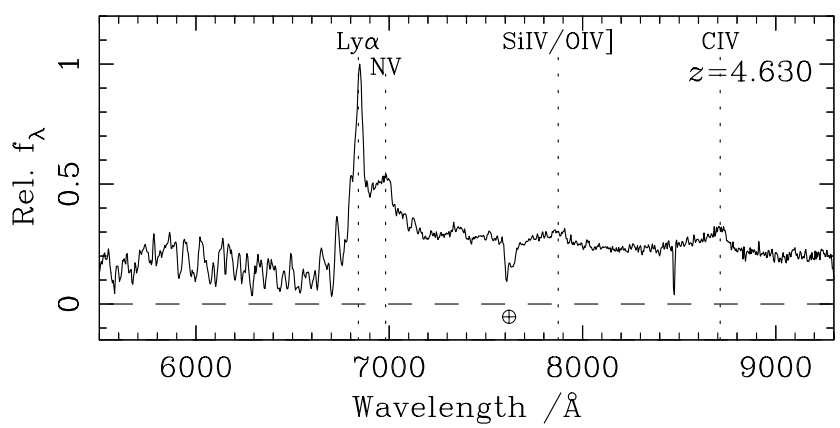

PMN J0326-3253

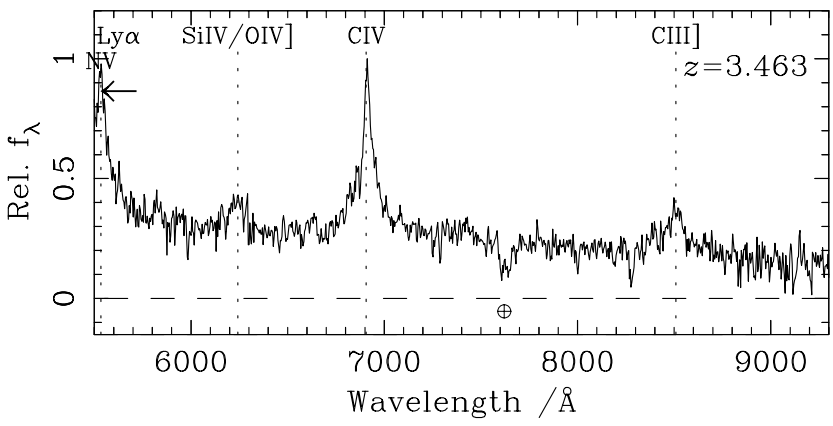

PMN J0525-3343

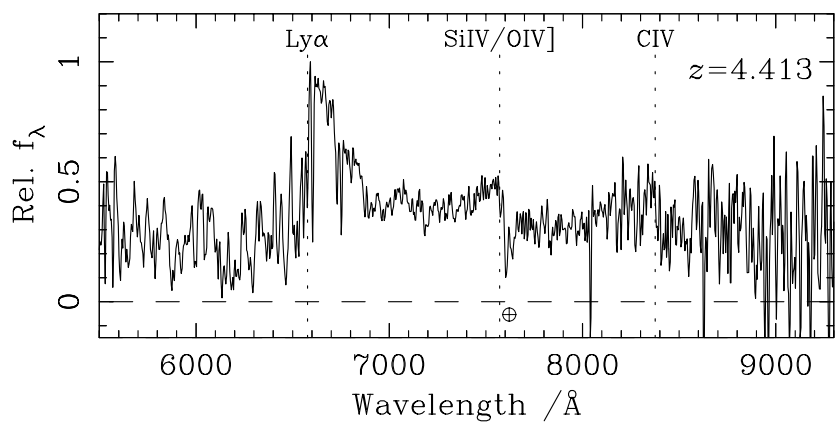

PMN J1043-2140

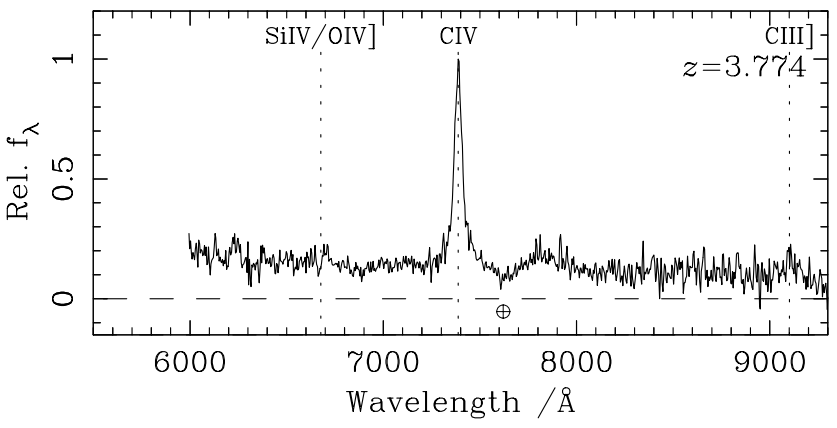

PMN J1108-1804

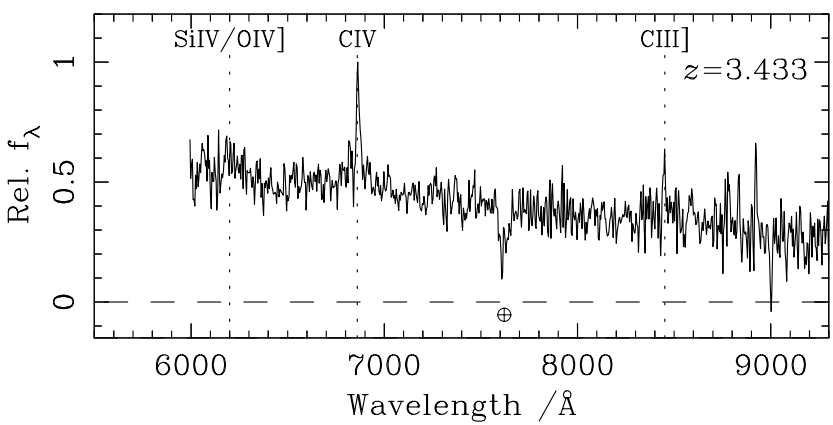

Fig. 5. Optical spectra of the $z>3$ quasars. 
PMN J1429-1616

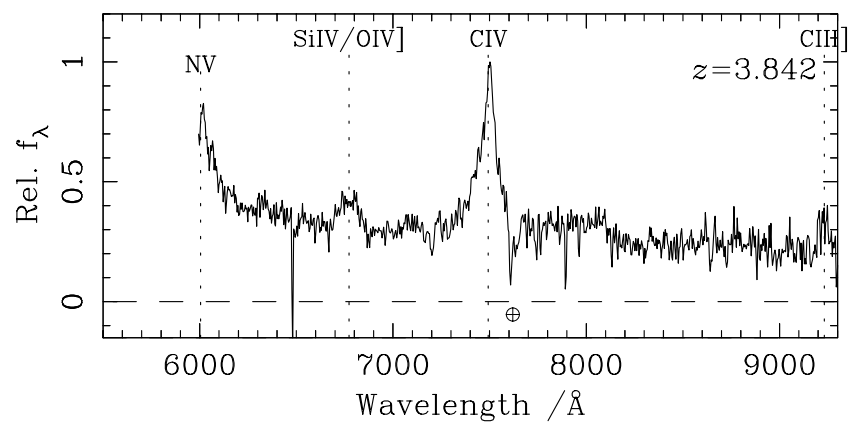

PMN J1451-1512

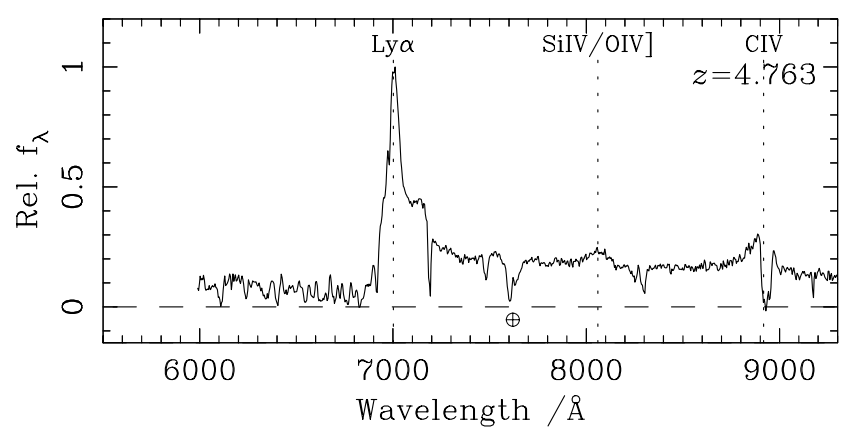

PMN J2042-2223

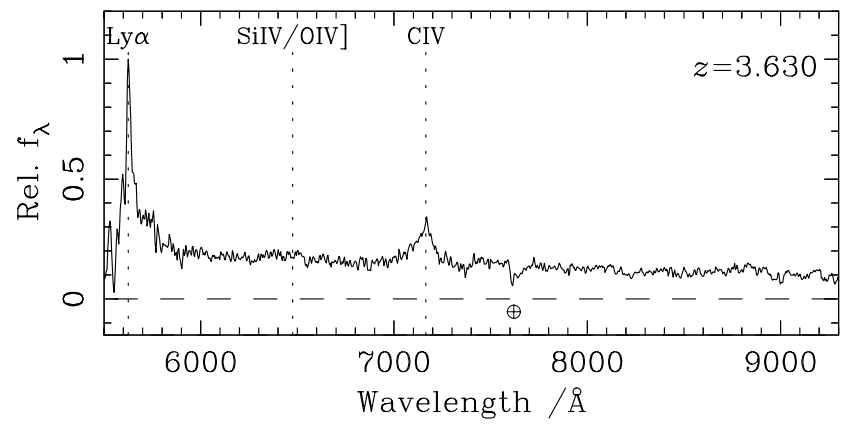

PMN J2134-0419

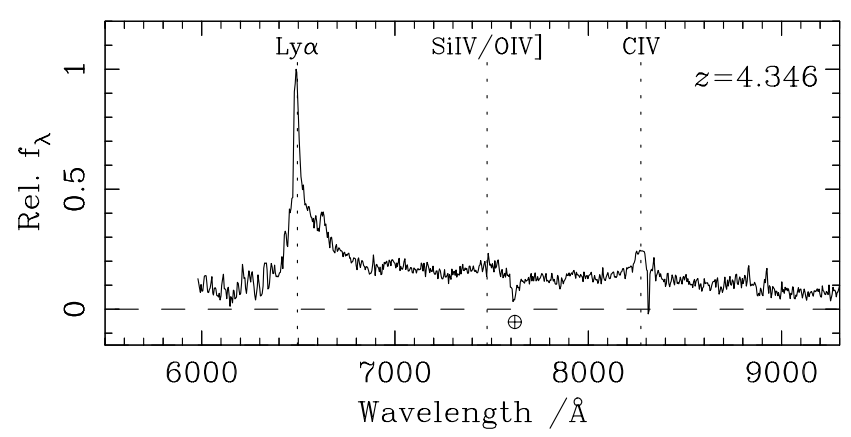

PMN J2219-2719

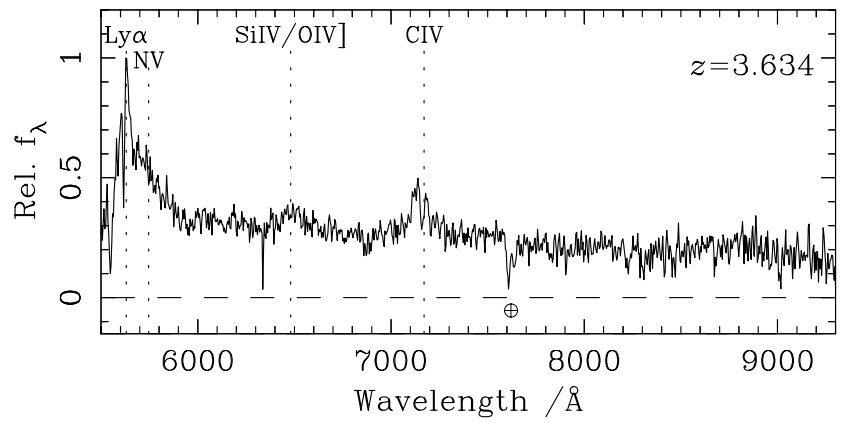

PMN J2220-3336

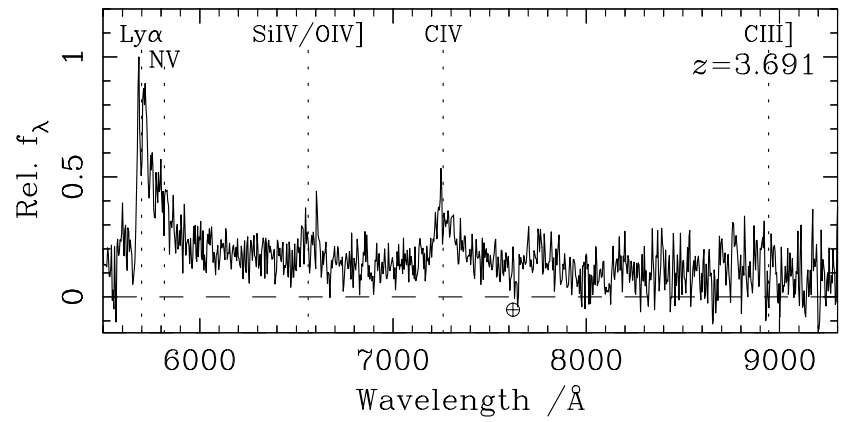

PMN J2314+0201

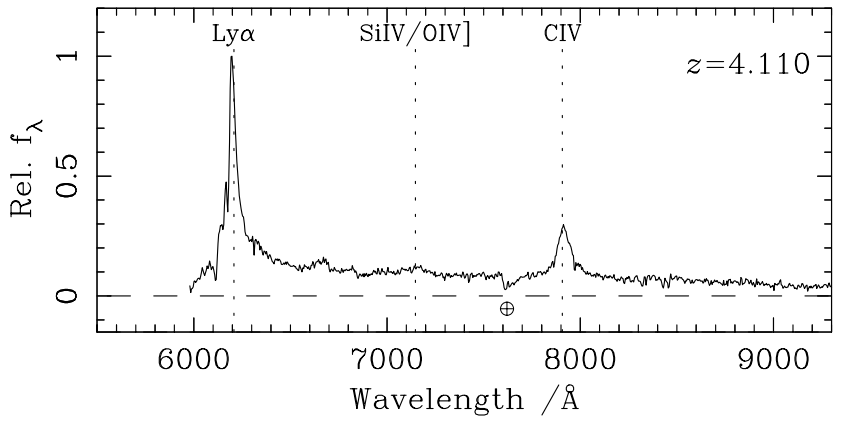

Fig. 5. continued. 
Table 3. Wavelengths of emission lines and redshifts for new $z>3$ objects, measured from the discovery spectra shown in Fig. 5. A $*$ indicates a line affected by absorption. Redshifts are calculated assuming rest wavelengths corrected for systematic shifts (Tytler \& Fan 1992) as follows:

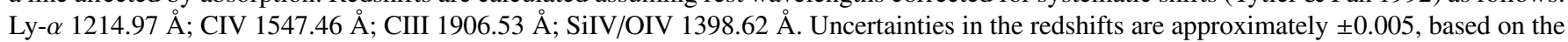
range of redshift determined from different emission lines in the same QSO.

\begin{tabular}{llllll}
\hline \hline Name & Ly $\alpha$ peak & SiIV/OIV & CIV & CIII & mean $z$ \\
\hline PMN J0022-0759 & 5956.46 & $6831.46^{*}$ & $7583.92^{*}$ & - & 3.896 \\
PMN J0214-0518 & 6053.03 & 6978.49 & 7714.46 & - & 3.986 \\
PMN J0235-1805 & 6455.15 & - & 8225.35 & - & 4.314 \\
PMN J0324-2918 & 6846.70 & - & 8703.26 & - & 4.630 \\
PMN J0326-3253 & 5422.49 & 6234.08 & 6913.65 & 8506.93 & 3.463 \\
PMN J0525-3343 & 6627.50 & 7536.03 & 8347.96 & - & $4.413 \dagger$ \\
PMN J1043-2140 & - & - & 7388.09 & 9101.68 & 3.774 \\
PMN J1108-1804 & - & - & 6863.86 & 8446.82 & 3.433 \\
PMN J1429-1616 & - & 6770.20 & 7498.27 & 9229.22 & 3.842 \\
PMN J1451-1512 & 7004.19 & 8057.75 & $8889.01^{*}$ & $10989.2 \dagger \dagger$ & 4.763 \\
PMN J2042-2223 & 5625.07 & - & 7166.39 & - & 3.630 \\
PMN J2134-0419 & 6488.28 & 7488.82 & 8269.41 & - & 4.346 \\
PMN J2219-2719 & 5628.22 & 6482.09 & $7138.42^{*}$ & - & 3.634 \\
PMN J2220-3336 & 5684.32 & 6570.76 & 7265.08 & - & 3.691 \\
PMN J2314+0201 & 6195.83 & 7153.88 & 7914.37 & - & 4.110 \\
\hline
\end{tabular}

$\dagger$ Péroux et al. measure $z=4.388$ for this QSO (when using the above rest wavelengths) based on a higher-quality spectrum. $\dagger \dagger$ Line measured from IR spectrum.

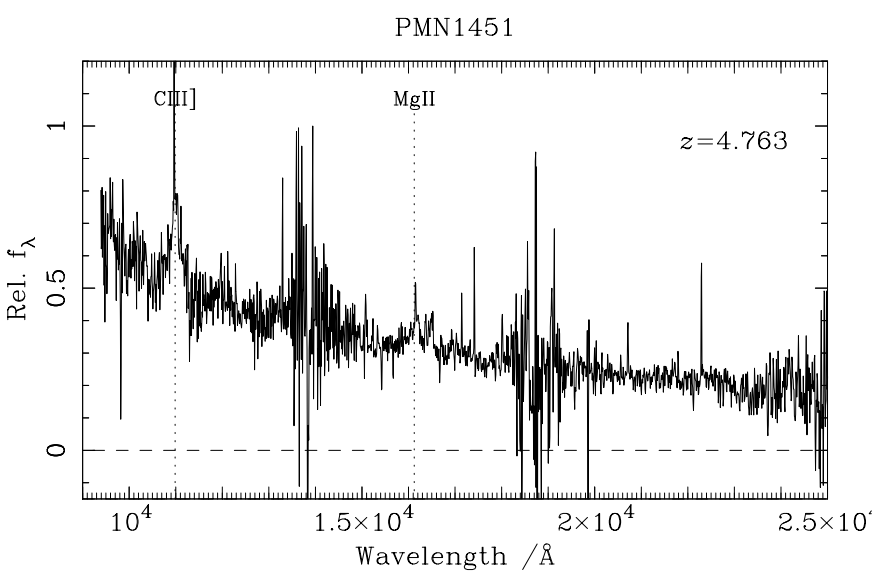

Fig. 6. IR spectrum of PMN J1451-1512, obtained at NTT.

\section{References}

Boller, T. H., Brandt, W. N., Fabian, A. C., \& Fink, H. H. 1997, MNRAS, 289, 393

Condon, J. J., Cotton, W. D., Greisen, E. W., et al. 1998, AJ, 115, 1693 Drinkwater, M. J., Webster, R. L., Francis, P. J., et al. 1997, MNRAS, 284,85

Dunlop, J. S., \& Peacock, J. A. 1990, MNRAS, 247, 19

Fabian, A. C., Celotti, A., Iwasawa, K., et al. 2001, MNRAS, 323, 373
Griffith, M. R, Wright, A. E., Burke, B. F., \& Ekers, R. D. 1995, ApJS, 97, 347

Hamuy, M., Suntzeff, N. B., Heathcote, S. R., et al. 1994, PASP, 106, 566

Hawkins, M. R. S., \& Veron, P. 1996, MNRAS, 281, 348

Hook, I. M., Becker, R. H., McMahon, R. G., \& White, R. L. 1998, MNRAS, 297, 1115

Hook, I. M., McMahon, R. G., Irwin, M. J., \& Hazard, C. 1996, MNRAS, 282, 1274

Hook, I. M., McMahon, R. G., Patnaik, A. R., et al. 1995, MNRAS, $273,63 \mathrm{~L}$

Kennefick, J. D., Djorgovski, S. G., \& De Carvalho, R. R. 1995, AJ, 110,2553

McMahon, R. G., \& Irwin, M. J. 1992, in Digitised Optical Sky Surveys, ed. H. T. MacGillivray, \& E. B. Thomson, Astrophysics and Space Science Library, 174 (Kluwer), 417

Péroux, C., Storrie-Lombardi, L. J., McMahon, R. G., Irwin, M., \& Hook, I. M. 2001, AJ, 121, 1799

Peterson, B. A., Savage, A., Jauncey, D. L., \& Wright, A. E. 1982, ApJ, 260, L27

Schmidt, M., Schneider, D. P., \& Gunn, J. E. 1995, AJ, 110, 68

Shaver, P. A., Wall, J., Kellermann, K. I., Jackson, C. A., \& Hawkins, M. R. S. 1996, Nature, 384, 439

Snellen, I. A. G., McMahon, R. G., Dennett-Thorpe, J., et al. 2001, MNRAS, 325, 1167

Tytler, D., \& Fan, X. 1992, ApJS, 79, 1

Warren, S. J., Hewett, P. C., \& Osmer, P. S. 1994, ApJ, 421, 412

Zickgraf, F.-J., Voges, W., Krautter, J., et al. 1997, A\&A, 323, L21 